\title{
Mitteilungen der DGINA
}

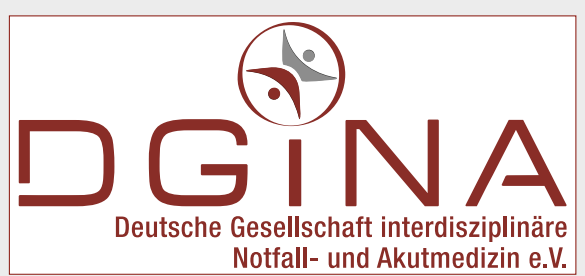

Notfall Rettungsmed 2020 $23: 238-239$

https://doi.org/10.1007/s10049-020-00704-9

(c) Springer Medizin Verlag GmbH, ein Teil von Springer Nature 2020
Deutsche Gesellschaft interdisziplinäre Notfall- und

Akutmedizin (DGINA) e.V.

Geschäftsstelle

Hohenzollerndamm 152 • 14199 Berlin

\section{Redaktion:}

Martin Pin (V.i. S.d.P.)

Florence-Nightingale-Krankenhaus

Kaiserswerther Diakonie

Kreuzbergstraße 79, 40489 Düsseldorf

Presse- und Öffentlichkeitsarbeit: Dr. Caroline Mayer

E-Mail: presse@dgina.de

\section{Wegen Corona-Krise: 3. Symposium der NRW- Landesgruppe am 28. März ging online}

Wegen der Corona-Krise konnte das für den 28. März geplante Symposium der DGINA-Landesgruppe Nordrhein-Westfalen nicht als Präsenz-Fortbildung stattfinden. Die Veranstalter entschieden sich deshalb kurzerhand, den Tag online durchzuführen.

Dr. Ingmar Gröning, der Gastgeber in Krefeld sein sollte, organisierte zusammen mit der Landesgruppe und der DGINAGeschäftsstelle eine Webkonferenz, die den Tag von 9.15 Uhr bis 16 Uhr eins-zu-eins abbildete. Die Veranstalter nutzten für das Webinar zwei Lizenzen von Now To Go und Medizin To Go. So konnte der virtuelle Raum in zwei Räume geteilt werden, in denen parallel je drei Sessions mit insgesamt 14 Referenten stattfanden.

Mit der Resonanz waren die Veranstalter sehr zufrieden. Während für die Präsenzveranstaltung bei Ingmar Gröning 140 Anmeldungen eingegangen waren, loggten sich bis zu 350 Teilnehmer gleichzeitig in das Online-Seminar ein. Neben Ärztinnen und Ärzten waren auch viele Mitarbeiter des Rettungsdienstes und Pflegekräfte dabei.

DGINA-Geschäftsführerin Karen Jerusalem, die die techni- sche Organisation übernommen hatte, resümiert: „Online-Veranstaltungen sind eine tolle Möglichkeit in Zeiten von Corona, die Teilnehmer fanden es auch alle gut." Natürlich müssten sich erst einmal alle an die Technik und die geänderten Bedingungen gewöhnen. So könne man sicher noch das Zeitmanagement besser im Auge behalten und die Teilnehmer mehr an die Hand nehmen. „Der Wechsel von einem virtuellen Raum in den anderen verlief etwas holprig, weil die Teilnehmer dazu verschiedene Browser nutzen mussten“, erklärt Jerusalem. „Aber viele haben das geschafft. Und von denen, die zwischendurch mal weg waren, sind zum Ende auch viele wiedergekommen."

Inhaltlich gingen zwei Sessions auch direkt auf die Corona-Pandemie ein. Der Virologe Prof. Dr. Jörg Timm der Uniklinik Düsseldorf hielt einen Vortrag zum Thema „SARS-CoV-2 Shut down or shut up!“. Dr. Norbert Pfeifer, Chefarzt aus Meran in Südtirol berichtete von den Abläufen in seiner Notaufnahme in der Ausnahmesituation. Daneben gab es u.a. Vorträge zu den Themen, „Der dementielle Patient in der Notaufnahme" von Irmgard Sicking, Dr. Sebastian
Casu Chefarzt der Notaufnahme Helios aus Salzgitter zu „Blutungsmanagement" und Martin Pin, Präsident der DGINA zu „COVID-19 eine Herausforderung für die Notfallmedizin“.
Da die Landesärztekammer 6 CME-Punkte für die Fortbildung bewilligt hatte, gab es zum Schluss noch einen Online-Test mit zehn Fragen, den Ingmar Gröning entworfen hatte.

\section{Hilfe in Belastungssituationen: SbE, PSU und EMPTY}

In der Corona-Krise gerät das medizinische Personal an die Belastungsgrenze. Wer Unterstützung sucht, um das Erlebte besser zu verarbeiten, oder sich über Möglichkeiten der Stressbewältigung informieren möchte, kann auf verschiedene Angebote zurückgreifen.

Die größte und älteste Einsatznachsorge-Organisation im deutschsprachigen Raum ist die SbE-Bundesvereinigung (Stressbearbeitung nach belastenden Ereignissen e. V.). Seit 1996 hat der Verein in seinen Schulungen über 130 regionale und organisationsinterne Teams ausgebildet. Deutschlandweit können derzeit über 110 Teams mit mehr als 1300 ehrenamtlichen Mitarbeitern bei Schadensereignissen alarmiert werden. Informationen zu den Teams, den Schulungen und Kontaktmöglichkeiten gibt es auf SbE-Webseite http:// www.sbe-ev.de.

Auf der Startseite der SbEWebseite ist auch ein COVID19-Merkzettel für Einsatzor- ganisationen und Vorgesetzte/ Einsatzleiter abrufbar. Darin wird beschrieben, welche Möglichkeiten der primären und sekundären Prävention Organisationen und Vorgesetzte bei Belastungsereignissen haben.

Der Münchner Verein PSUAkut bietet schon seit Jahren Stressbewältigungsseminare und Peer Support für verschiedene Berufsgruppen aus dem Rettungswesen an. Seit Ausbruch der Corona-Pandemie können auf der Webseite zusätzlich InfoBlätter zur Selbsthilfe heruntergeladen werden. Beispielsweise „Möglichkeiten zur Stressreduktion für medizinisches Personal (COVID-19)“, „Möglichkeiten der Stressreduktionen im Quarantänefall“ oder „Informationen zur Stressreduktion für medizinische Führungskräfte (COVID-19)“. Download unter www.psu-akut.de -> Hilfreiche Informationen.

Für alle Mitarbeiterinnen und Mitarbeiter im bayerischen Gesundheits- und Rettungswesen 
hat PSU-Akut außerdem eine telefonische Helpline eingerichtet. Unter der Nummer 089545584 40 bekommen Anrufer aus Bayern täglich von 8 bis $21 \mathrm{Uhr}$ Unterstützung bei besonderen Belastungs- und Stressreaktionen im Rahmen der Corona-Pandemie.

Weiterhin gibt es auch das Projekt EMPTY der Young DGINA, das mit der SbE und mit PSU-Akut kooperiert. EMPTY steht für „Emergency Medicine Problem Talk der YoungDGINA“. Konkret bedeutet das: Vier Mitglieder des DGINA-Nachwuchses bieten jeden Montag zwischen 16-19 Uhr ehrenamtlich eine Peer-Beratung am Telefon an (Telefonnummer: 0171-1274658). In bis zu zwei Gesprächen wird die Belastung, die zum Anruf führte, strukturiert besprochen. Wenn gewünscht, gibt es auch eine Beratung zu anschließenden Versorgungsmöglichkeiten. Die Ansprechpartner der YoungDGINA, die die Telefonanrufe entgegennehmen, sind geschulte Peers. Sie haben ein entsprechendes Fortbildungs-Modul beim Münchner Verein PSUAkut absolviert.

Zielgruppe von EMPTY sind vor allem junge Ärztinnen, Ärzte und Pflegekräfte. Anrufen kann aber grundsätzlich jeder, der eine belastende Situation erlebt hat und darüber reden möchte. Das Gespräch ist anonym und vertraulich. Wer am Montag zwischen 16 und 19 Uhr keine Zeit hat, kann auch eine spezielle Terminanfrage mit Rückrufnummer anempty@dgina.de schicken.

\section{David-Williams-Award 2020: Vorschläge für Preisträger gesucht}

Auch in diesem Jahr vergibt die DGINA wieder den David-Williams-Award für besondere Leistungen und Verdienste um die Notfallmedizin in Deutschland.

DGINA-Mitglieder können jetzt bereits begründete Vorschläge an die DGINA-Geschäftsführerin Karen Jerusalem richten - bevorzugt per E-Mail: kontakt@dgina.de. Über die Vergabe entscheidet der DGINAVorstand. Der Preisträger erhält eine Goldmünze im Wert von ca. 2500 Euro.

Die Deutsche Gesellschaft für interdisziplinäre Notfall- und Akutmedizin DGINA e.V. ehrt mit dem - nach dem britischen Notfallmediziner David Williams benannten - Award herausragende Leistungen in der fachübergreifenden Notfallmedizin. Dr. David Williams war vor fünfzig Jahren maßgeblich an der Einführung eines Facharztes für
Notfallmedizin beteiligt. Damit legte er einen der Grundsteine für die Professionalisierung der notfallmedizinischen Versorgung. Der Preis soll an diese herausragende Leistung erinnern.

Mit Dr. Matthias Brachmann wurde 2014 zum ersten Mal einen Nicht-Mediziner ausgezeichnet. Weitere Preisträger waren die langjährigen federführenden Schriftleiter der Zeitschrift „Notfall + Rettungsmedizin“ Prof. Dr. Hans-Richard Arntz und Prof. Dr. Uwe Kreimeier, Dr. Werner Wyrwich, Vorstandsmitglied der Berliner Ärztekammer, die frühere EuSEM-Präsidentin Dr. Barbara Hogan, die OnlinePlattform NOW TO GO (Kurzform für NOtfall-Wissen TO GO) und der Magdeburger Chirurg Prof. Dr. Felix Walcher.

Der Preis wird auf der diesjährigen Jahrestagung der DGINA in Wolfsburg verliehen. 